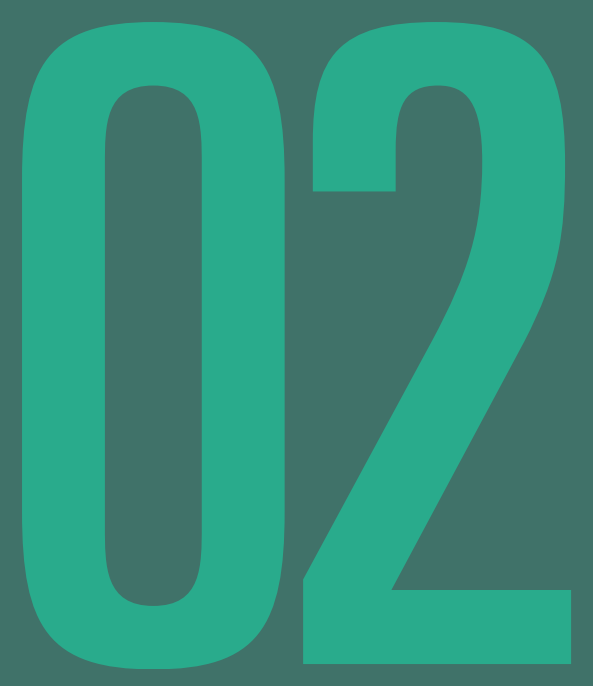

\title{
LA INTELIGENCIA
}

ARTIFICIAL Y LA

ALTA COSTURA

El caso del "cognitive dress"

Artificial intelligence and high fashion

The cognitive dress case

LTI. Mayela Maricela Villagrán Loa'

mayev177@gmail.com

LDI. Vladimir Villa Guerrero ${ }^{2}$

vg_vladimir_1994@hotmail.com

Universidad Autónoma de Nuevo León. Estudiantes en la Maestría en Ciencias con Orientación en Gestión e Innovación del Diseño. Nacionalidad Mexicana 
Palabras clave: Inteligencia Artificial, Alta Costura, Tendencias, Consumo Keywords: Artificial Intelligence, Haute Couture, Trends, Consumption.

Resumen:

Las innovaciones en el ámbito de la moda se vuelven cada vez más disruptivas desde que comenzó el siglo $X X I, y$ esto se debe al actual apoyo no solo de expertos en la materia sino de disciplinas ajenas a dicha industria. Estas definiciones van desde el uso de materiales sintéticos vanguardistas en las prendas de las pasarelas más afamadas, hasta en la manera en como se difunden, se comportan o se hacen llegar a las poblaciones (y la postura que toman ante ellas), también y por otra parte se presentan incursiones de tecnologías de punta como la Inteligencia Artificial, que no eran imaginables en un pasado, presentando un panorama nuevo y redituable a la sociedad actual. La intervención en procesos de manufactura y diseño por parte de algo tan complejo como la biónica es algo que ocurre ya en distintos proyectos como los que se han hecho en interacción entre la Inteligencia Artificial y las casas de Alta Costura. En el presente artículo se indaga sobre un caso particular donde inteligencia artificial y su proceso de diseño se muestran.

\section{Abstract}

Innovations in the field of fashion have become increasingly disruptive since the beginning of the twentieth first century, and this is due to the current support not only of experts in the field but disciplines outside the fashion industry. From the use of avant-garde synthetic materials in the garments of the most famous catwalks, the way in which they spread, behave or reach the masses (and the position they take upon them), till the incursion of cutting-edge technologies, that were not imaginable in the past, present a very promising and profitable panorama to the current society. The intervention in manufacturing processes and design by something as complex as Intelligent Artificial is something that already occurs in different projects such as those that have been made by the interaction between Artificial Intelligence and Haute Couture houses. In the present article, a particular case of a collaboration of this type is investigated. 
Mayela Maricela Villagrán Loa

\section{INTRODUCCIÓN}

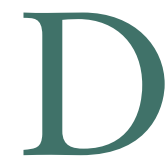

emos un pequeño vistazo a la creación tecnológica, nos permite ver los años 40 cuando empezaron a funcionar las primeras computadoras llevando consigo al principio de la Inteligencia Artificial y siendo reconocida como una disciplina de las ciencias tecnológicas surgiendo a partir del año 1956. El campo de la inteligencia artificial, o IA, va más allá: no solo intenta comprender, sino que también se esfuerza en construir entidades inteligentes. donde se intentaba aplicar un razonamiento artificial de un sistema para que esta interactuara con una persona. (Russell y Norvig, 2004).

En un principio se les llamo "cerebros electrónicos o cerebros tecnológicos” (Gómez Herrera, s.f.) ya que empezaron realizando sumas y operaciones simples sin imaginarse lo que llegaría a ser.

Actualmente en el siglo XXI, la Inteligencia Artificial es representada de diversas formas sea como humanoides, robots, sistemas informáticos de lenguajes complejos, y donde estos realicen funciones que se pensaban eran solo de los humanos y destinados a realizar tareas o actividades que los seres humanos hacen, efectuándolos de una manera mucho más avanzada y rápida. 
Este proceso es denominado "razonamiento inteligente" (Russell y Norvig, 2004). y está basado en el aprendizaje automático que significa el análisis de los datos, circuitos electrónicos y lenguajes avanzados de computadora, que busca imitar la red neuronal del ser humano y que ésta logre aprender.

En la actualidad nuestra cultura ha ido cambiando, haciendo una inclusión de estos sistemas que día a día nos ayudan y acompañan en nuestras actividades y vida cotidiana, esta interacción es llamada "interacción hombre - máquina inteligente ${ }^{1}$ " porque nos ayudan en la toma de decisiones utilizando el "sentido lógico" que les ayuda a actuar y pensar facilitando nuestras necesidades.

Y no solo en la cotidianidad del día a día nos pueden ser útiles sino también es una gran herramienta en las industrias y empresas globales de talla internacional. A lo que nos referimos con esto y podemos notarlo con facilidad, es como se integra la Inteligencia Artificial en la industrial de la moda, siendo más específicos en la Alta Costura.

El término de la alta costura hace referencia la creación de piezas para vestir, que son únicas y confeccionadas a la medida del cliente y es acuñado en el año 1700 por Marie-Jeanne Bertín mejor conocida como Rose Bertín, quien fue la sombrerera y modista de María Antonieta, y quien ayudó a que la reina tuviera un estilo muy propio en esa época en Francia.

A lo largo de la historia múltiples marcas han surgido para llevar al mundo sus creaciones, algunas de estas marcas son: Dior, Channel, Louis Vuitton, Hermès o Versace teniendo detrás a los más exitosos diseñadores como Yves Saint Laurent, Pierre Cardin y André Courrèges entre otros. Hoy en día sólo existen 14 casas de alta costura reconocidas en el mundo y sólo 800 personas según la Féderation Française de la Couture están en posibilidad o tienen oportunidad económica de adquirir este tipo de prendas, entre las que figuran desde celebridades hasta miembros de la realeza como lo son Carolina de Mónaco; su cuñada, Charlene, Christine Lagarde y millonarios rusos, chinos o árabes. En 2010, el 30\% de los compradores de Alta Costura procedían de los Emiratos Árabes Unidos.

1 Interacción Hombre - Maquina Inteligente: Es la mejora en la calidad del trabajador en si ambiente de trabajo, acrecentando la eficacia funcional de las actividades a realizar. 
Este particular tipo de moda se ve exhibido actualmente en eventos de élite y premiaciones a lo largo del mundo como lo son los Oscares, el Festival de Cannes o eventos a beneficio, asistidos por celebridades como lo es la MET Gala, que se realiza cada año y es organizada por Anna Wintour, la editora en Jefe de la aclamada revista internacional de modas Vogue, siendo este un evento de los más significativos para la sociedad de la Haute Couture ${ }^{2}$, ya que contribuye a la exposición de moda anual del Instituto del Vestido del Museo Metropolitano de Arte en la ciudad de Nueva York; los costos de admisión giran alrededor de 238 mil dólares por persona, y se recaudan aproximadamente 9 millones de dólares al finalizar el evento.

La MET Gala se caracteriza por la temática o diferenciación en cada una de sus ediciones eligiendo temas de interés actual y que puedan llevar a un debate en la sociedad.En el año 2016 la temática elegida fue “Manus x Machina: La Moda en la Era de la Tecnología”. La elección de este tema tiene que ver con el despunte de la Inteligencia Artificial y la Tecnología ya que poco a poco estaban entrando al mercado; las celebridades invitadas llevaron modelos de Alta Costura y las diversas prendas incluyeron las últimas innovaciones tecnológicas que estuvieron a su alcance como lo fue en el caso de la casa de moda italiana Marchella.

Marchella tuvo la oportunidad de trabajar en conjunto con la Inteligencia Artificial y el resultado de esta fusión fue el "Congnitive Dress"

La MET Gala, data del año 1948, y tuvo lugar en el Rainbow Room del Rockefeller Center N.Y., donde se le llamó en varios medios la fiesta del año. Su coordinación estuvo a cargo de Eleanor Lambert, una publicista de moda de la época, seleccionada por el Instituto del vestido. No fue hasta el año de 1972 donde empezó a llamar mayormente la atención de la sociedad, cuando la consultoría especial del Instituto estaba a cargo de Diana Vreeland, quien organizó los reconocidos tributos a Balenciaga, Yves Saint Laurent y a "La Gloria del Vestido Ruso" por nombrar algunos, colaborando con personas importantes de la época, como Jacqueline Kennedy Onassis, la entonces Primera Dama de los E.E.U.U. (The Costume Institute, 2018).

2 Haute Couture: Termino francés que se refiere a la creación de prendas exclusivas a la medida del cliente. 
Aunado al tema de la Gala mencionado en la introducción, y a su temática de Manus x Machina: La Moda en la Era de la Tecnología, bajo el mando de Anna Wintour, pudo apreciarse en la alfombra roja, cómo es que se fusionaron dos temas completamente distintos de una manera disruptiva dando como resultado diseños de moda creados con ayuda de la Inteligencia Artificial y un claro ejemplo de eso es la intervención de Watson-IBM, un procesador de última generación que almacena la inteligencia artificial desarrollada por IBM desde el año 2011. Fue hasta entonces que la compañía decidió empezar a hacer pruebas sencillas de sus capacidades como el responder a preguntas generales y básicas, y al hacerlo de una manera satisfactoria la Inteligencia Artificial Watson, empieza a ser alimentada con bases de datos internas de la compañía, lo que le facilitaba aprender y tener acceso a la información que se le fuera solicitada de una manera fácil, concreta y rápida.

De esta manera es como Watson poco a poco empezó a tomar relevancia y al ser aceptado en diferentes campos; uno de éstos, la industria de la moda, co-diseñó el ya previamente mencionado Cognitive Dress. Marchella se dio a la tarea de proporcionar a Watson - IBM toda la información sobre el diseño que se llevaría a cabo. El procesador fue alimentado con los datos de las tendencias de la época en cuanto a materiales, colores, siluetas, acabados y procesos de diseño que ayudaron a llegar al resultado final.

El vestido fue creado con leds que cambiaban de color en respuesta a las "emociones" de tweets, que Watson se encargaba de analizar y procesar durante el evento, donde cada color representa una emoción traducida por Watson.

La Inteligencia Artificial marca una pauta de hacia dónde podríamos llevar la fusión con actividades como la industria de la moda, brindando un panorama extenso y prometedor de lo que se puede llevar a cabo con estas colaboraciones, que hoy en día son más comunes. 


\section{LA POSTURA DE LA SOCIEDAD ANTE LA INDUSTRIA DE LA ALTA COSTURA}

Se puede observar cómo las tendencias que marca la alta costura, en los eventos antes mencionados se impregna en los elementos cotidianos de los estratos más comunes en la sociedad, que más objetivamente se traducen en vestimenta, productos, artefactos decorativos, e incluso a cosas más subjetivas como guiones o atmósferas en películas, inclusive en canciones, libros, y otros factores que rodean a las masas. Bertuzzi (2016: 19), señala que "la moda se ve influenciada directamente por los hechos sociales, económicos, políticos, culturales, así como también artísticos, la sociedad y el contexto en el que se encuentre también se ven influenciados por los mandatos de la moda”. Bajo este argumento se defiende que es una relación en la que ambas variables (sociedad y moda) coexisten, y se presenta un continuo flujo de información donde puede diluirse, o ser percibido de forma imprecisa; qué variable depende de cuál.

Uno de los casos en los que la Moda, o más precisamente el Haute Couture dicta los patrones de comportamiento, no solo de la industria textil, o de productos, sino estilos de vida; es cuando se mezcla con la producción masiva, como sucedió en el año 2015, cuando Balmain (una de las 14 casas reconocidas de Alta Costura) hizo una colaboración que consistió en el diseño de 110 piezas que se venderían en la multinacional tienda sueca de H\&M, que entra dentro de la categoría de Prét-a-portér1 y esto solo intensifica el ideal aspiracional al que se ven sometidos los bloques sociales, intentando imitar algo que no está en sus posibilidades (en este caso particular, económicas) alcanzar.

Desde siglos pasados, la exposición de lo que tiene un bloque social contra lo que carece el otro, en la eterna lucha de clases sociales que propone Karl Marx en el Materialismo histórico, aquel que se encuentre bajo la sombra del más fuerte siempre va a querer poseer lo que no tiene o lo que se le niega. Aterrizando esta teoría a la postura actual de la sociedad ante la alta costura, una vez conceptualizadas las ideas de los pioneros en estilos, las marcas comercializan de forma masiva mediante el procedimiento de educar por medios persuasivos como lo son programas de televisión, producciones cinematográficas, revistas y más recientemente mediante conectores o el fenómeno de los influencers en las 
redes sociales, siendo estos últimos la epítome del principio aspiracional, donde se introduce la idea a la persona de querer ser como alguien más, a quien admira o posee un estilo de vida que le gustaría llevar.

Como ejemplo concreto de lo anterior tenemos lo que se presenta en el contexto de los festivales musicales, como lo son el Lolapalooza (llevado acabo en Norte y Sudamérica) e inclusive más famosos como lo es el Coachella (Californa, E.E.U.U.), donde se emiten reportes de estudios y tendencias por empresas como lo es la WGSN, que dictan las pautas clave (llamadas Key Fashion Trends ) que debe seguir una marca, si quiere posicionarse bien en el mercado durante las siguientes temporadas.

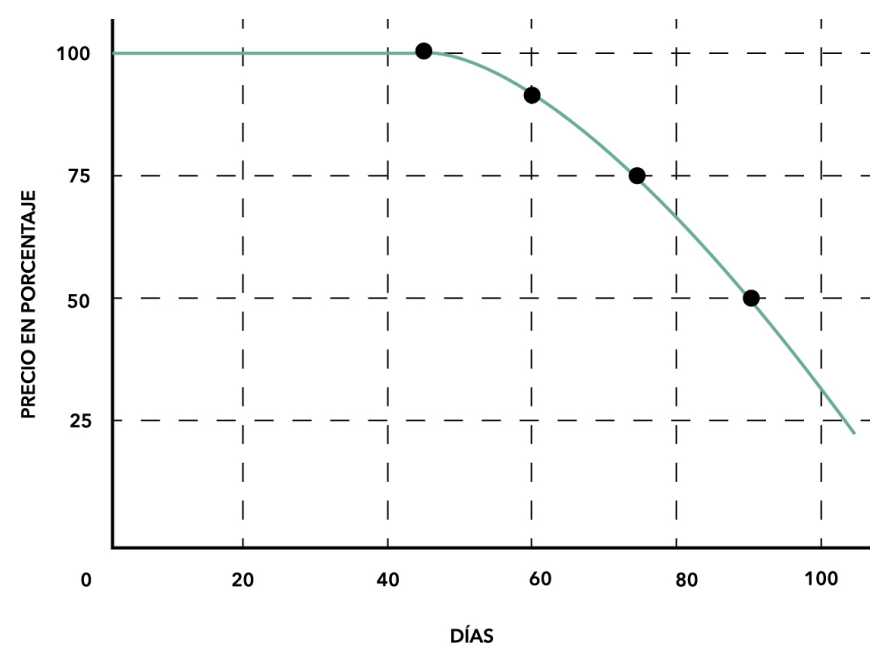

Figura 4. Diagrama elaborado por el autor

El ritmo al que la gente debe consumir la moda se ha visto acelerado de una manera exponencial desde que inició el siglo XXI, donde las prendas se insertan en el mercado a su precio original por 45 días, después de esto hasta el día 60 aproximadamente se ofertan con $10 \%$ de descuento, hasta el día 75 con el $25 \%$, en el día 90 puede llegar a ofertarse hasta el $50 \%$ y todo lo que no se venda después se lleva a lo que las tiendas llaman Remate/Sale.

Este sistema estimula a los compradores a adquirir más prendas incluso aunque no las necesiten, una vez más por la influencia que reciben de los medios persuasivos, que hablan directamente a las necesidades de afiliación e integración de la entidad individual en los diversos círculos sociales. 
Dicho esto, y retomando eventos como la MET Gala, es aquí donde las casas de alta costura marcan las pautas de cómo deberá ser la tendencia de moda que llega hasta las manos de los consumidores con menos poder adquisitivo y que también se someten a la dinámica de consumo acelerada, previamente mencionada.

\section{CONCLUSIONES}

La moda desde sus inicios fue un diferenciador para las personas, ya que con la fusión de los diferentes campos se demostró que se pueden obtener resultados relevantes y funcionales como lo fue el Cognitive Dress y que no solo eso sino que también con la ayuda de la inteligencia artificial se pueden optimizar los procesos de diseño ya que el análisis de los datos se vuelve más preciso y rápido.

Poco a poco iremos avanzando a tal grado de que algo como la Inteligencia Artificial Watson, pueda estar al alcance de todos para colaborar no solo en la industria de la moda sino también en el día a día con las empresas de la industria tecnológica.

Los eventos como la MET Gala, son una excelente oportunidad para dar inclusión de las nuevas tecnologías, por las que usualmente no se opta por falta de recursos, ya que, al ser un evento dirigido a personas con alto valor pecuniario, esto no se vuelve un impedimento, las hace participes en los diseños y los dotándolas de un aire fresco, prospectivo y vanguardista.

La incursión de Watson nos ayuda a tomar de las redes sociales un sin fin de interpretaciones, ayudando a codificarlas y convertirlas en sentimientos o emociones humanas de las cuales puede llegar a aprender para mejorar; así fue como el Congnitive Dress nos habló de lo que el mundo pensaba y decía sobre él, representando los comentarios digitales de las redes sociales y haciendo tangibles cosas tan subjetivas como las emociones

El pensar en soluciones que incursionen a futuro, es una forma de cómo las empresas o las productoras de diseño (no sólo de modas) se pueden colocar de manera altamente retribuible en el mercado, ya que el 
primero en generar innovaciones suele ser el que coloquialmente "pone las reglas del juego", es decir establece o dictamina pautas de cómo deben ser los productos que se ofertan a los consumidores.

Es valido que como en el caso de Marchella e IBM, dos industrias que a simple vista no están relacionadas puedan desarrollar metodologías o proyectos donde se les permita trabajar juntas, reforzando la multidisciplinariedad o trabajo conjunto de las ciencias con las humanidades y las artes, e impulsando nuevas ideas o realidades materiales en los alcances de las creaciones Humano-Máquina Inteligente y donde lo que hace unos años pareciera algún guion de ciencia ficción, hoy es una realidad, y más que eso (en beneficio del sistema de consumismo actual) es una realidad.

Dado lo anterior, puede concluirse que las fronteras entre lo técnico y lo estético (las máquinas inteligentes y la alta costura, por ejemplo), se diluyen aceleradamente al paso del tiempo, volviéndose sistemas cada vez más complejos, y el factor catalizador para que esto suceda es la sinergia que se da entre la imaginación humana y lo lejos que nos puede llevar la tecnología de punta. 


\section{REFERENCIAS}

Gómez Herrera R. (s.f.). La inteligencia artificial ¿Hacia dónde nos lleva?. México: ¿̇cómo ves? Revista de Divulgación de la Ciencia de la UNAM. Recuperado de http://www. comoves.unam.mx/numeros/articulo/2/la-inteligencia-artificial-hacia-donde-nos-lleva.

Russell, S. J. y Norvig, P. (2004). Inteligencia Artificial. Un enfoque moderno Segunda edición. Madrid: Pearson Educación.

The Costume Institute. (2018). The Metropolitan Museum of Art. Recuperado de www.metmuseum.org/about-the-met/curatorial-departments/the-costume-institute

Bertuzzi M. (2016). Moda, cultura e identidad: el vinculo entre la moda y la sociedad. Recuperado de http://fido.palermo.edu/servicios_dyc/publicacionesdc/archivos/633_libro.pdf 